\title{
Placement of applications in computing clouds using Voronoi diagrams
}

\author{
Caroline Shouraboura • Pavel Bleher
}

Received: 23 October 2010 / Accepted: 5 September 2011 / Published online: 23 September 2011

(C) The Brazilian Computer Society 2011

\begin{abstract}
The vision of millions of users launching tens of millions of applications running on millions of globally scattered servers presents a new challenge for Cloud Providers: how to assign so many virtual applications to physical servers, while meeting latency needs, improving network utilization, and satisfying availability constraints. Today's application placement puts too much burden on the cloud user, lacks scalability and inhibits the global reach of Public Clouds.

The size, breadth, and dynamic nature of Public Clouds present a special challenge to the task of placement. Cloud Providers able to provide rapid decisions and frequent optimizations for placement will have significant competitive advantage. Not only will they provide the best customer experience, their costs will be lower as they make more efficient use of their network resources. However, given the calculations required, data structures and the algorithms used to process location-based decisions must be as globally scalable as the Public Clouds themselves.

In our study, we define a novel data structure, the Virtual Cloud Model, for modeling global cloud resources. We adapt a well-known geometric device, Voronoi Diagrams, and combine it with near-real-time network latency information. We then solve the application placement problem and suggest an API for Cloud Providers to support both "low-latency" and "high-availability" applications. The algorithms are scalable, parallelizable and distributable.
\end{abstract}

\section{Shouraboura}

Forest Ridge, 9927 171st Ave SE, Newcastle, WA 98059, USA

e-mail: carolinesh@forestridge.org

P. Bleher $(\bowtie)$

Indiana University-Purdue University Indianapolis, $402 \mathrm{~N}$.

Blackford Street, Indianapolis, IN 46202, USA

e-mail: bleher@math.iupui.edu
Keywords Cloud computing - Application placement . Virtual cloud model · Voronoi diagram

\section{Introduction}

The vision for Cloud Computing is to have millions of users launching tens of millions of applications running on millions of globally scattered servers. This presents a new challenge for Cloud Providers, which requires new data structures and scalable algorithms for placing and managing user applications.

Behind the Public Cloud is a physical network of data centers with millions of servers, hidden from us through virtualization. Virtualization offers Public Cloud providers the flexibility to assign each application to run on any of its available physical servers. The primary variables in deciding placement of an application within the Public Cloud are (1) minimizing latency (and therefore bandwidth consumption) of the compute resource with respect to points on the network with which it must communicate (other compute resources, persistence layers, and endpoints outside of the cloud are a few examples) and (2) availability constraints specifying a minimum separation compute resources must have from other resources to avoid correlated failure.

Current research in this area is nascent both because of cloud computing's relatively brief history and the breadth and complexity of applications and their needs. One important classification of the problem has been contributed by [1], where applications with users spread across a global user community can be optimized by placing data optimally with respect to their use. This paper deals with the vast set of applications having close binding with their data such that both data and application can be treated as a single "Virtual Machine" (VM). Because of the complexity of different 
underlying application architectures and assumptions about data locality, both the Volley approach described in [1] and the approach we describe here should be useful for those building cloud applications and providing cloud infrastructure. A data-oriented approach to placement can and should coexist with an application-oriented approach.

In this paper we choose latency as the driving variable because it is a characteristic of the physical layout of the cloud, dictated by such things as speed of light, network device limitations, and the network cabling connecting compute resources. Bandwidth is a parallel variable, but is generally highly correlated with latency [25]. We discuss a case where bandwidth is an independent constraint in the Future Work section. In practice cloud providers face many more restrictions such as capacity and cost of system resources, cost of relocating Virtual Machines (VMs) to remote sites, disaster recovery, committed vs. uncommitted quality of service, and others. In this paper we propose two extensions to our algorithm for applying geography and capacity constraints.

Traditional techniques for managing application placement lack the scalability required by Public Clouds [16, 32]. A large-scale private data center might contain tens of thousands of servers running well-understood applications statically allocated to servers. Even if the private data center is virtualized, the numbers of servers involved and the slow rates of change limit issues of scale. Public Clouds today are rapidly growing to, and beyond, millions of servers scattered across different countries and continents, running dynamically changing application mixes. Many applications automatically scale up by requesting more resources from the Cloud and then scale down by releasing resources, as demand subsides. Frequently, applications must communicate with each other, whether for synchronizing game state, exchanging intermediate computational results, assembling web pages, or querying databases. The network in a Public Cloud supplies substantial network bandwidth in support of cross-application communication.

For a group of applications requiring communication with each other, placement proximity on the underlying physical servers is critical. Cloud Providers have a strong incentive to keep communication paths as short as possible, because the cost of provisioning bandwidth increases with distance. Latency also increases significantly with distance, so customers of a Cloud receive a better experience with shorter network paths. In order to find two or more servers which are "close" to each other, it would seem necessary to examine the latencies between all pairs of servers in the Cloud to compute an optimal placement. The calculation of latencies must be executed frequently given the network is a shared resource and is therefore subject to regular changes in performance. This would mean a running time of $\mathcal{O}\left(n^{2}\right)$, where $n$ is the number of servers in the cloud. Public Clouds might have millions of servers running tens of millions of applications owned by different companies and individuals. This kind of scale requires a high-performance algorithm capable of running in near-real time.

In this paper we describe a viable model of the Cloud precomputed in $\mathcal{O}(n \log n)$ time and a scalable $\mathcal{O}(k+\log n)$ algorithm to solve the application placement problem, where $k$ is the number of servers required by the application and $n$ is the number of servers in the Cloud. The model is based on Voronoi Diagrams.

\section{Voronoi diagrams}

In this paper we use a 2-dimensional Voronoi Diagram computed for a set of $n$ points on a plane. It is defined as follows.

Definition 1 The set of all points closer to a given point in a point set than to all other points in the set is the Voronoi Polygon for the point. The collection of all the Voronoi Polygons for a point set is called its Voronoi Diagram.

The dual graph to the Voronoi Diagram (Fig. 1) is a Delaunay Triangulation (Fig. 2). By definition, the Delaunay Triangulation contains an edge connecting two points if and only if their Voronoi regions share a common edge. The Delaunay Triangulation $\boldsymbol{D T}(\boldsymbol{P})$ for a set $\boldsymbol{P}$ of points in the plane possesses a remarkable property such that no point in $\boldsymbol{P}$ is inside the circumcircle of any triangle in $\boldsymbol{D T}(\boldsymbol{P})$. This property characterizes a $\boldsymbol{D T}$ uniquely [29]. Shamos and Hoey [31] described a divide-and-conquer algorithm to compute the Voronoi Diagram in $\mathcal{O}(n \log n)$ time. Subsequently, many other usable and efficient algorithms and data structures were discovered [2] and implemented for computing Voronoi Diagrams [3, 4, 10, 13, 24, 27]. Voronoi Diagrams found their application in many different fields, including computer science [3, 4, 12, 24, 26, 27].

Public Clouds have a new problem of optimizing the cost and experience of tens of millions of simultaneous applications running on a network of millions of servers distributed world-wide. In our study we tackle the novel problem of carving up the space within a Computer Cloud. Our approach allows rapid allocation of a set of virtual machines for each application that reflect low latency and bandwidth, reducing the consumption of physical network paths in the Cloud. Our method is based on the idea that the more applications communicate with each other, the "closer" they need to be placed in the Cloud. Our research consists of three steps. First, we will define a Virtual Cloud Model (VCM), a novel, efficient structure to model the Cloud. Second, we will describe two algorithms for assigning applications to servers using the VCM and suggest an API for Cloud Providers. Lastly, we will describe the process of Cloud defragmentation to maintain healthy application placement in the Cloud. 
Fig. 1 An example of a

Voronoi diagram

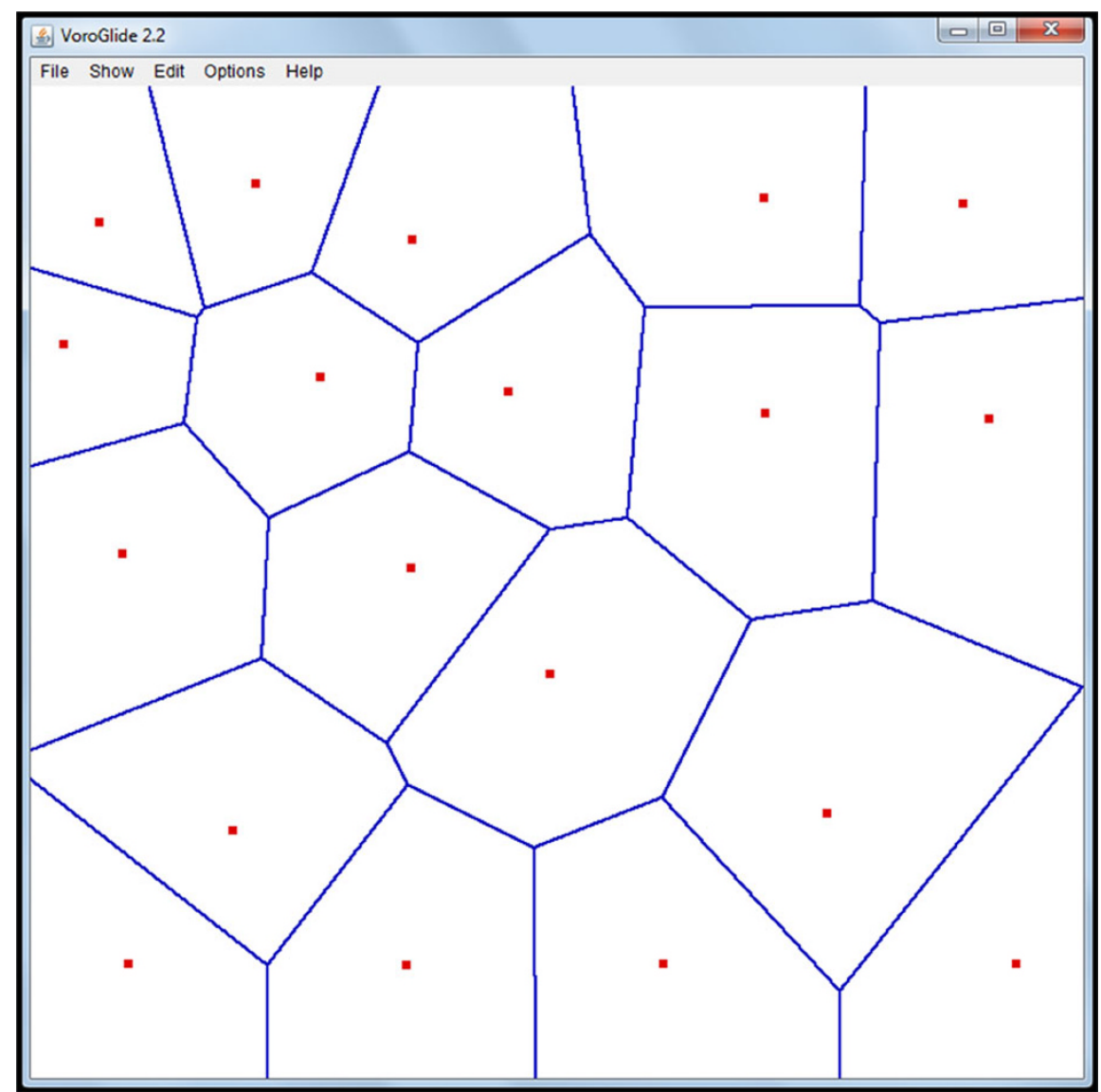

\section{Virtual cloud model (VCM)}

To reduce the network congestion and latency in communication between applications, we would like to position connected applications "close" to each other. Several properties of Voronoi Diagrams make it a relevant and appealing structure for Cloud partitioning: the linear size of the Voronoi Diagram, the proximity information stored in the Voronoi Diagram in an explicit and computationally useful manner and its localized and easily distributable behaviors. This allows us to store and perform operations on Voronoi Diagrams locally, utilizing the distributed computing Cloud environment.

Let $\{\boldsymbol{R}\}$ be a set of all server racks and $\{\boldsymbol{r}\}$ be a set of all routers, switches, load balancers, and other traffic related network devices in the cloud (Fig. 3). The main objects of our model are server racks, but we are also going to include other devices to reflect the actual physical network. Let $\{\boldsymbol{D}\}$ be the union of $\{\boldsymbol{R}\}$ and $\{\boldsymbol{r}\}$, representing all relevant devices in the Cloud. Let us assume that each device has an IP address, which is uniquely mapped to its geographic location using the function Location $(\boldsymbol{D})$. The geographic location of a given device is a good approximation of its network "closeness" to other devices. To best economize cost and latency, Cloud Providers keep physical cabling and network distances at a minimum, making physical location a reasonable first-order approximation of network closeness.

For each device $\boldsymbol{D}$ we call function $\operatorname{Location}(\boldsymbol{D})$ and establish a set of points on the plane, representing geographical coordinates of all server racks and routers on the network. We then perform Voronoi tessellation (Fig. 4) on the points and obtain a Geographic Delaunay Triangulation (Fig. 5) using VoroGlide software [34]. While the VoroGlide algorithm was sufficient for our study, a parallel algorithm described in [21] could be better suited for the distributed architecture of the Cloud. We use the precomputed Geographic Delaunay Triangulation as the foundation for building a VCM. We still need to address two important challenges of modeling the Cloud: first, network traffic between servers is routed inconsistently with respect to physical geography due to network engineering factors such as distances of cabling/fiber runs, over-subscription of bandwidth between switches and routers, and routing weights for cost and redundancy; and second, the "distances" effectively change as networks become more or less congested.

To address the challenge of considering dynamic network performance, we measure and incorporate the actual network latency into the model. To avoid measuring network latency between each pair of devices, which would require an $\mathcal{O}\left(n^{2}\right)$ operation, we limit the measurements to the edges 
Fig. 2 Dual Delaunay triangulation

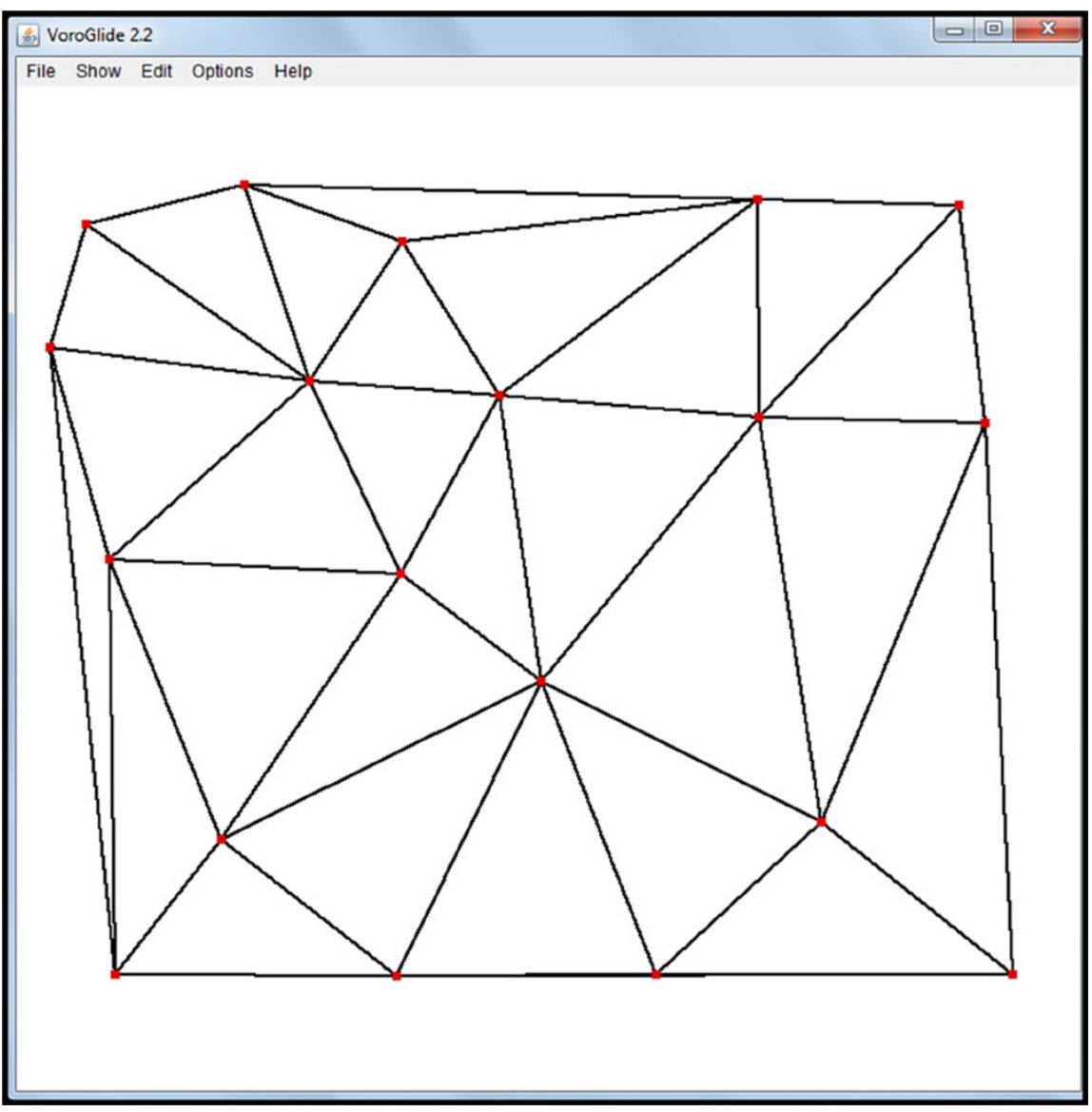

of our precomputed Geographic Delaunay Triangulation. It is important to note that the number of edges in Delaunay Triangulation is $\mathcal{O}(n)$. For every edge we capture its latency, defined as the time it takes for a packet of information to travel between its vertices in milliseconds. A variety of network monitoring tools could be used to perform this task. A Cloud Provider with access to the physical devices would simply be able to run the probe from each of its devices directly. We then assign weight $w_{l}$ to every edge of Geographic Delaunay Triangulation equal to the network latency of the edge. The resulting structure is the Virtual Cloud Model (VCM), which we are going to use next for application placement. Note that this diagram is not a physical representation of how network packets move across the network; rather it reflects the relative latency servers have with respect to each other.

For scaled up implementation, VCM could be distributed and the weight of edges could be dynamically updated as the latency of the network changes due to congestion. Using VCM, we can now define the "distance" between devices in the network.

Definition 2 The distance between device $\mathbf{D}_{1}$ and device $\mathbf{D}_{2}$ in the VCM is the sum of weights of all edges on the shortest path between $\mathbf{D}_{1}$ and $\mathbf{D}_{2}$.

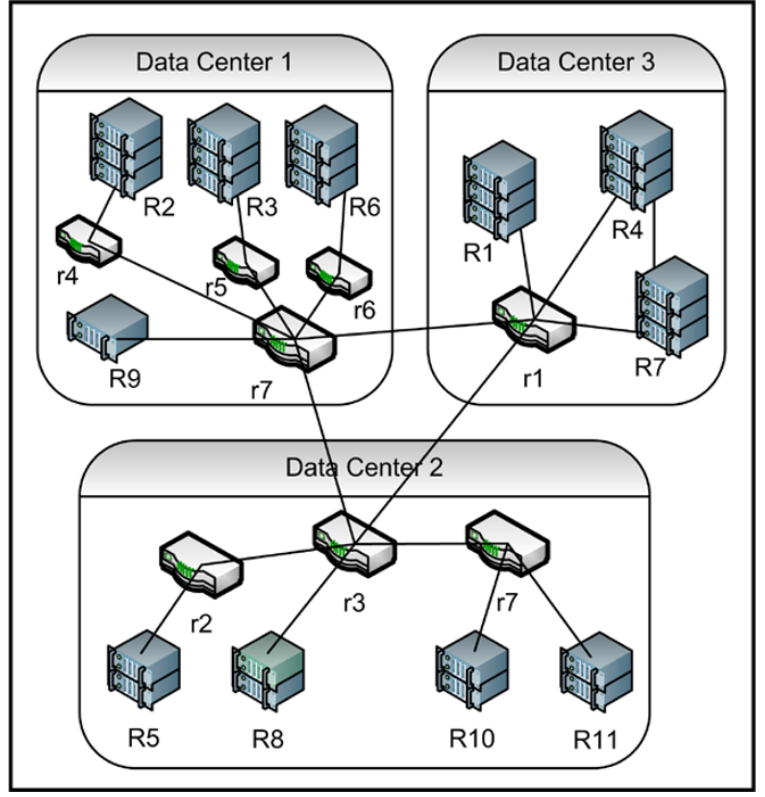

Fig. 3 An example of racks and routers in the cloud

Because over-subscription of network devices which aggregate network traffic creates performance bottlenecks, Cloud Providers are using network architectures that reduce 
Fig. 4 Geographic Voronoi diagram

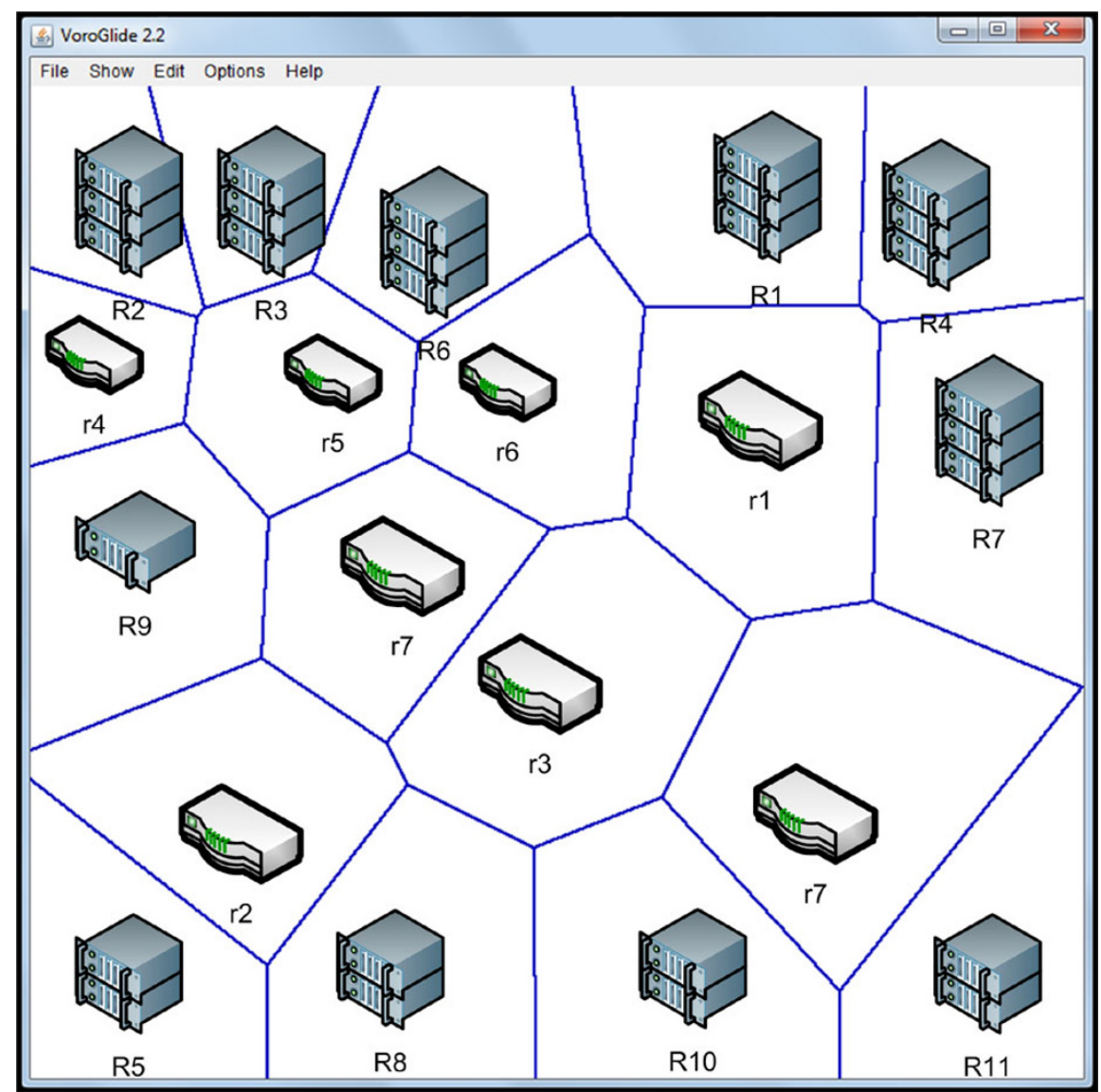

the impact of these devices on the performance of the network. Based on this, we decided to use a simplified model, which excludes routers, switches, load balancers and other traffic related network devices.

In our simplified Virtual Cloud Model we only consider server racks, and we perform the Voronoi tessellation for the set $\{\boldsymbol{R}\}$ of all server racks and we obtain a Geographic Delaunay Triangulation for the set $\{\boldsymbol{R}\}$. The algorithm performs in $\mathcal{O}(n \log n)$ time and requires $\mathcal{O}(n)$ space [10], where $n$ is the number of server racks. We capture the weight of each edge as a latency of each edge of Delaunay Triangulation for $\{\boldsymbol{R}\}$ (Fig. 6).

The well-explored algorithmic space of Delaunay Triangulation provides us with readily available methods for efficiently dealing with changes in resource capacity and location, such as rack addition or failure. Methods for efficiently recalculating only a local portion of the triangulation are described in [15]. Paper [19] also describes algorithms for distributing a Delaunauy Triangulation across many separate computers.

To illustrate VCM using a very small subset of a reallife Cloud, we selected 12 servers on Amazon's Cloud in 12 different locations: four servers in Europe running in different locations, one server in Asia, in Singapore, one server on the US West Coast in San Francisco, and six servers on the US East Coast in different locations. We then estimated server locations based on the region information, provided by Amazon, and constructed Geographic Voronoi Diagram for 12 servers shown by points in Fig. 7 and corresponding Geographic Delaunay Triangulation (Fig. 8) using VoroGlide software [34].

We then used "ping" to dynamically capture the actual network latency between server racks for every edge of Geographic Delaunay Diagram. Ping is a computer network utility used to measure the round-trip time for packets sent from the local host to a destination computer in milliseconds (ms). We constructed the VCM for 12 servers as an illustration for our model. For every edge of the Delaunay Triangulation we assigned the weight $w_{l}$ equal to the average roundtrip time between the servers, divided by 2 , in milliseconds (see Fig. 9). Our illustration in Fig. 9 also highlights the opportunity to improve our algorithm by considering VCM on a globe. For example, the latency between AP1 (Asia) and USW1 (San Francisco) is less than the latency between AP1 (Asia) and EU4 (Europe), which correlates with distances on the sphere. Since VCM is designed to work for a global Cloud, we could extend our model to construct Geographical Voronoi Diagram on a sphere. In [23], the authors show 
Fig. 5 Geographic Delaunay triangulation

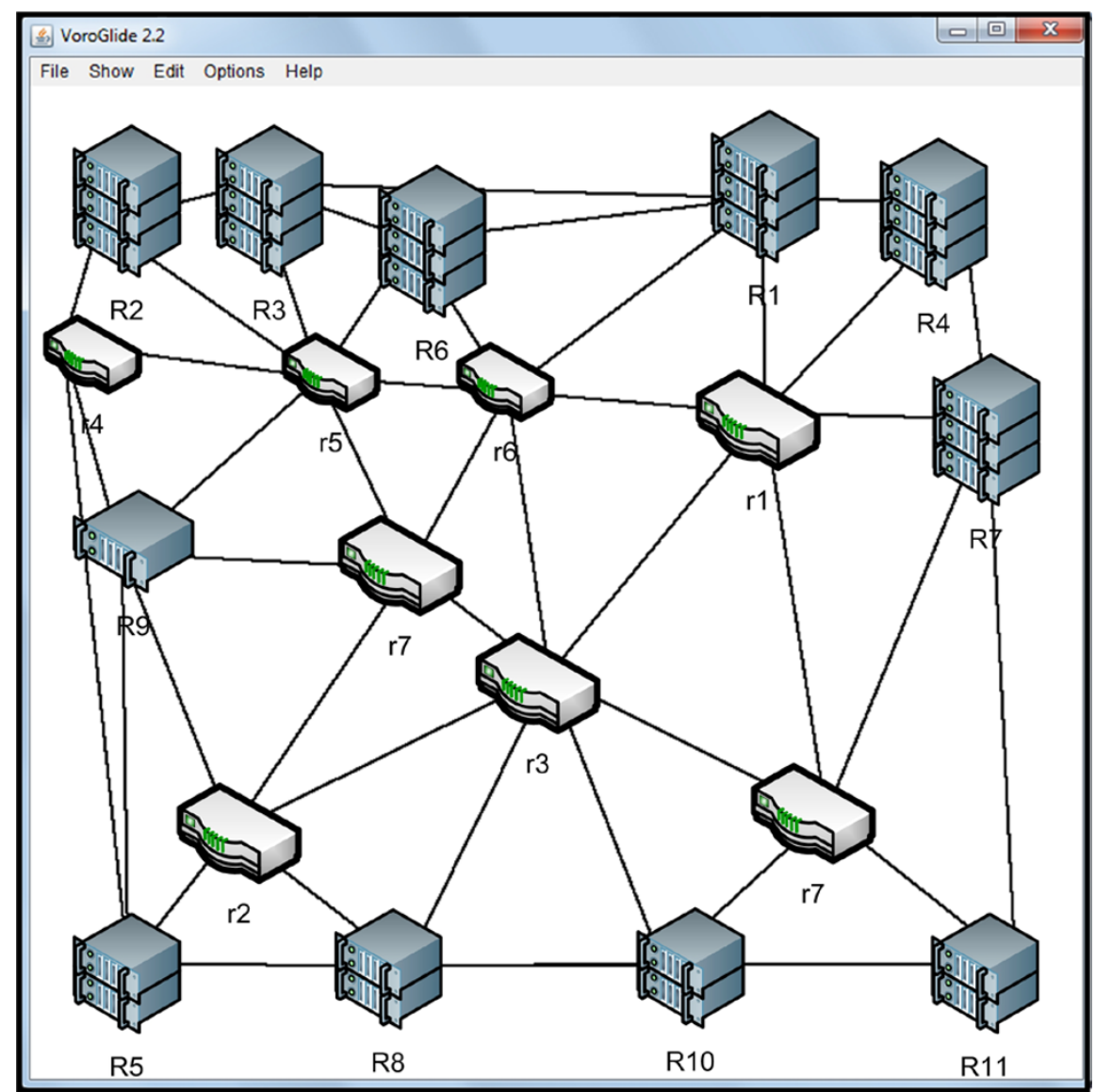

that for a given a set of sites on a sphere, their spherical Voronoi Diagram can be derived by computing two planar Voronoi Diagrams of suitably transformed sites in the plane.

\section{Placing applications in the cloud using VCM}

When a user launches an application in the Cloud, the user first defines a virtual machine image specifying the software that will be run upon startup. The user then requests a single or multiple virtual machines from the Cloud via an API call. The Cloud Provider allocates a set $\left\{V_{j}\right\}$ of requested virtual machines and then launches a copy of user-defined software image on each virtual machine, thus providing the user with a set of running "instances". Typically there are many instances running simultaneously to provide scalability of an application. For example, in an online gaming application a virtual "world" will be hosted on many instances of the game's application. This is important because no single server could possibly have enough capacity to serve all users of the game. Each user will be assigned to a particular virtual machine instance. Instances will communicate state information with each other in order to keep the "world" consistent in appearance to all participants. When a user launches an application, the number of instances required is specified as an API parameter. These instances can be placed anywhere in the cloud, however, some locations are better than others stemming from application requirements. For example:

1. If an application requires a frequent communication with computers outside the cloud, it is better to place application instances close to user's anchor location $U$.

2. If an application requires frequent communication among instances, it is important to place those instances close to each other to reduce latency in communication and avoid performance degradation. In those cases the proximity of application placement would also benefit the cloud provider by reducing the consumption of network resources.

3. Some applications require increased availability. In many cases failures happen on a cluster of servers located in close physical proximity. Examples of such failures are a failure of one rack, which brings down all servers in that rack, a failure of a row of racks due to router failure, or a failure of a data center, bringing down many rows of racks, due to a power outage or other major malfunction. To ensure that parts of an application continue running 
Fig. 6 An example of

simplified virtual cloud model with weights

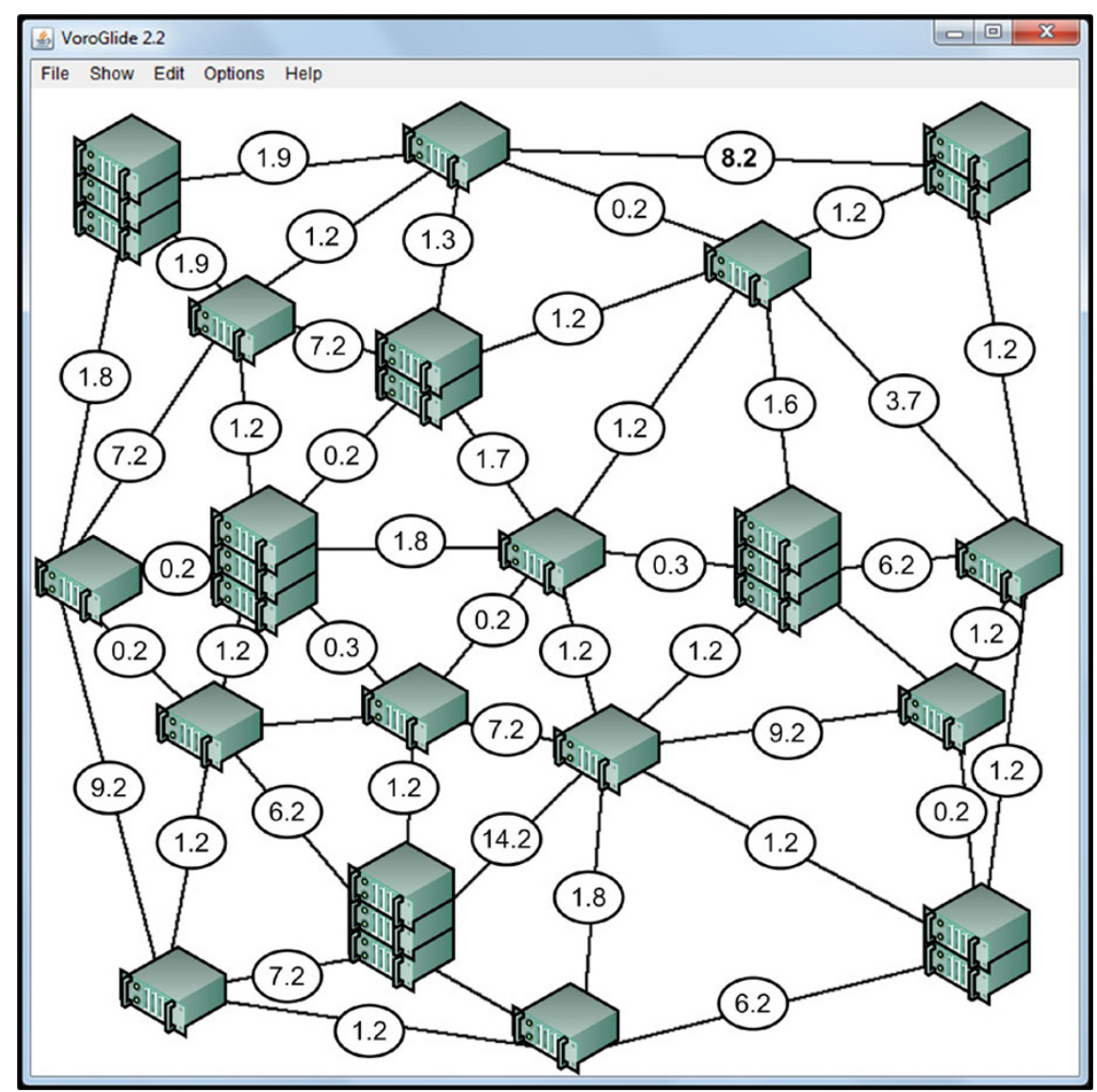

Fig. 7 Geographic Voronoi diagram for 12 servers

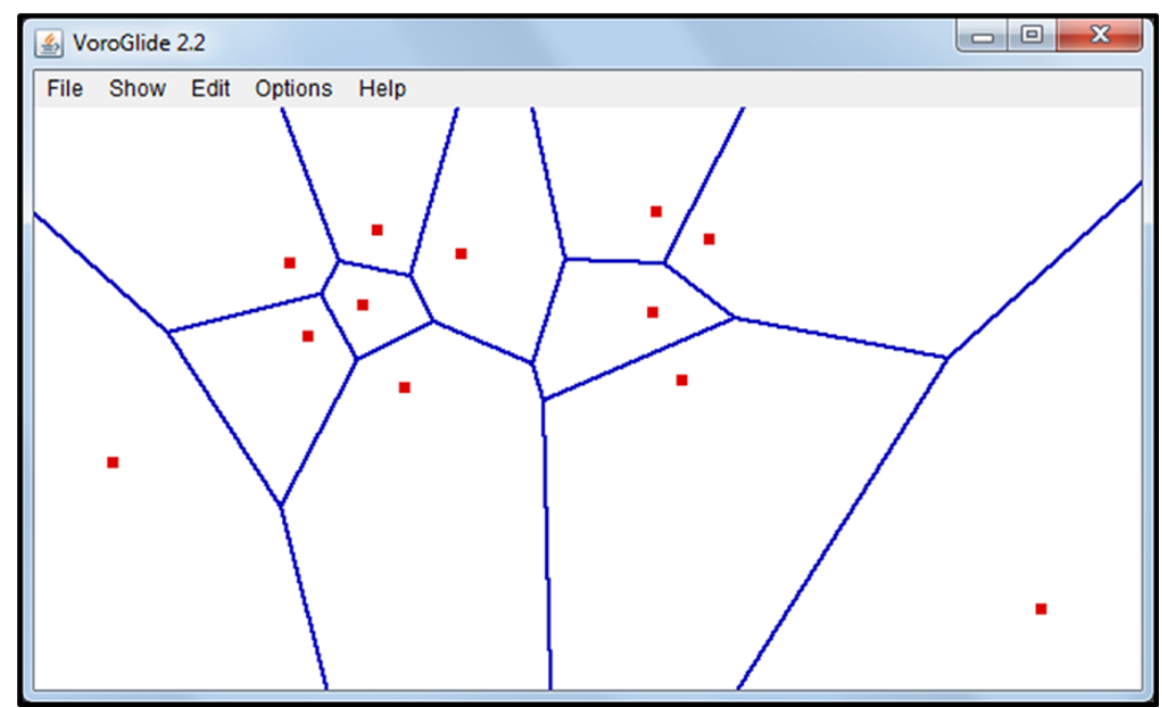

under those scenarios, it might be desirable to space the application across remote servers to increase availability at the price of latency.

Given the variety of application placement requirements, the Cloud Provider could offer customers an API to specify the desired application placement strategy. The conceptual API for a launch request of $k$ instances could look like:

Launch $(k:$ number,

$U$ : location, A : application,

$L$ : latency, $S$ : separation); 
Fig. 8 Dual geographic Delaunay triangulation

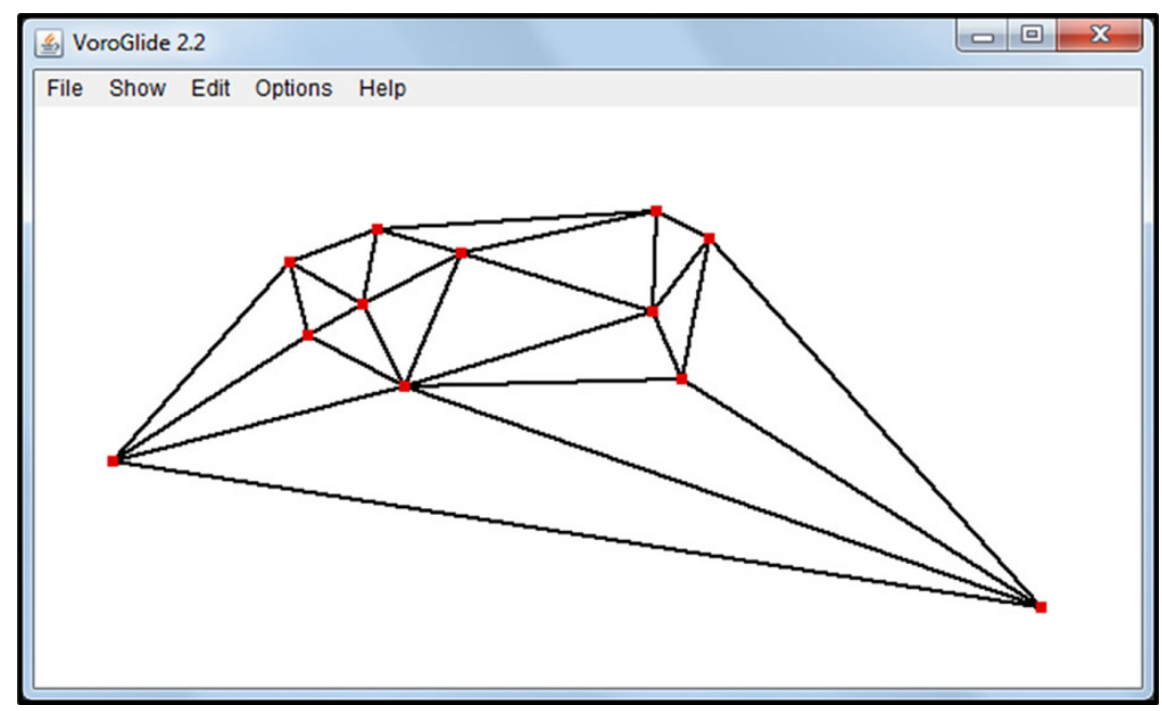

Fig. 9 An illustration: VCM for 12 servers

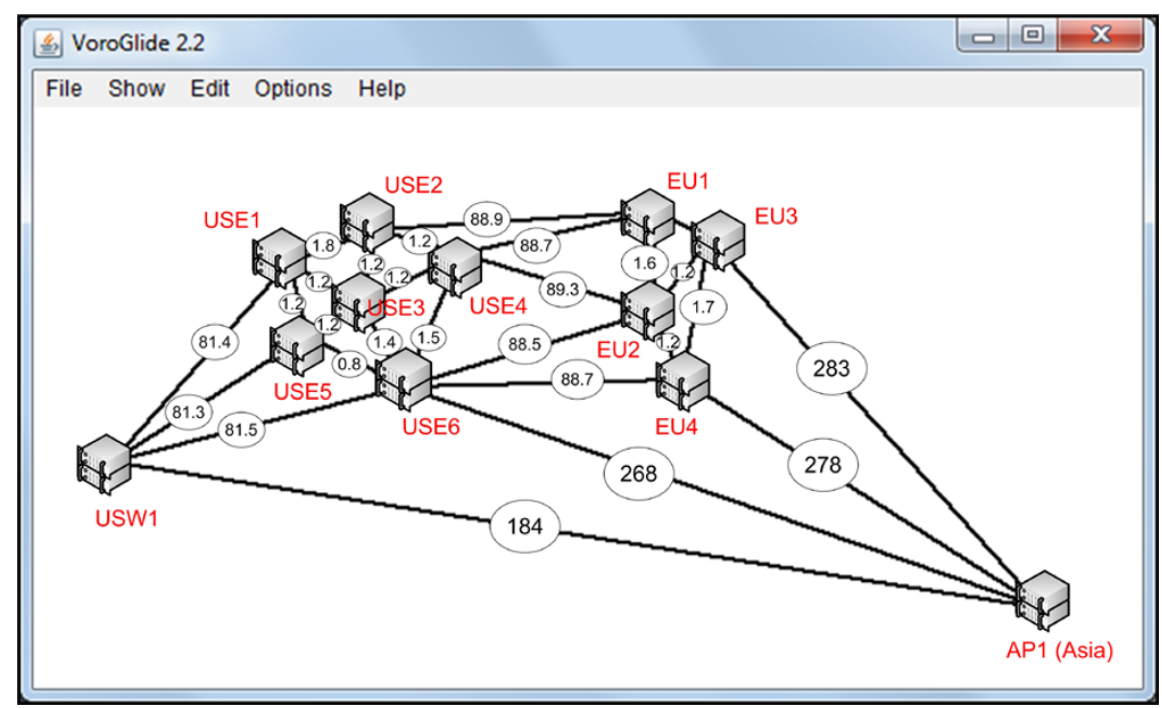

We propose to use VCM for the implementation of different placement strategies.

\subsection{Latency constraint}

The following algorithm uses VCM to place instances close to a user's location and to each other. Let us assume that a user defined a desired software image $I$ and requested $k$ instances running the image $I$, launched in proximity to his location $U$. We first use the VCM to find the triangle with three server racks as vertices containing $U$. There are several wellknown algorithms to perform this search in $\mathcal{O}(\log n)$ time (see [9] and references therein). Once we found the triangle containing $U$, we consider its three vertices and server racks associated with them and select the server rack $R_{i}$ closest to $U$.

We then allocate virtual machines on $R_{i}$ to run user applications until we exhaust the capacity of $R_{i}$.
Capacity can be measured and optimized in a number of ways here, the variety of which is discussed in the Future Work section below. For the purposes of this algorithm, we assume that the virtual machine we are placing has an application capacity which can be compared to the capacity available on $R_{i}$. We then perform a breadth-first search of VCM graph to find required capacity, looking for the closest servers to $R_{i}$. This placement algorithm reduces latency and bandwidth consumption, for a given application, placing parts of the application on neighboring servers by traversing the VCM and taking into the account actual, near-real-time network latency.

1. Put all neighbors of $R_{i}$ in a list.

2. Examine all server racks in the list and select the server rack $R_{j}$ closest to $R_{i}$ using the definition of the "distance" provided above (as the sum of all weights on the shortest path). 
3. If $R_{j}$ has available capacity then allocate available capacity of $R_{j}$ to our application.

4. Add all neighbors of $R_{j}$ to the list.

5. Remove $R_{j}$ from the list.

6. Repeat steps $2-5$ until we find requested capacity to launch $k$ instances to run the application.

The algorithm performs in $\mathcal{O}(k+\log n)$ time. We first find the closest server rack to user location $U$ in $\mathcal{O}(\log n)$ time and then we traverse the VCM to place $k$ instances in $\mathcal{O}(k)$ time. Here we make an assumption that the degree of each vertex in the VCM graph is bounded by a constant independent of $n$. This assumption looks plausible but it requires an additional verification in concrete Computing Clouds. In the Poisson-Voronoi probabilistic model, in which the sites are random points from a standard Poisson process on the plane (see, e.g., the paper [11] of Hinde and Miles and the monograph [22] of Møller), there are various theoretical and numerical results supporting the assumption. As shown in the classical paper [20] of Meijering, the mathematical expectation of the number $N$ of the site neighbors in the Poisson-Voronoi model is equal to 6. Calka [6] derives an exact formula for the probability $p_{k}$ that $N=k$ as a complicated $2 k$-dimensional integral, but as the author remarks himself, the integral is very difficult for an asymptotic analysis as $k \rightarrow \infty$. Numerical results of Tanemura [33], Brakke [5], and others, strongly indicate that $p_{k}$ decay exponentially as $k \rightarrow \infty$, and $p_{k}$ is well approximated by a generalized gamma distribution. Therefore, the estimate $\mathcal{O}(k)$ for the time to place $k$ instances in the Poisson-Voronoi model looks very reasonable.

\subsection{Availability constraint}

For applications wishing to minimize latency, the aforementioned algorithm alone may be applied. However, minimizing network distance typically increases the probability of correlated failure. For example, two virtual machines placed on the same physical machine have extremely low latency but obvious correlated failure cases. The same virtual machines placed on different physical servers in the same rack still typically depend on a common network switch and power path. While power and network redundancy can be implemented within servers and racks at increased expense, even separation of virtual machines across racks within a data facility leaves exposure to correlated failure due to unexpected events. Examples include room cooling malfunction, failure of automatic power transfer switches, unanticipated ground faults, and network route misconfiguration. Different application architectures require different tradeoffs between latency and availability. High-performance computing applications bias entirely toward latency and will generally desire placement within the same rack. Missioncritical business applications or backup solutions may require geographic separation. VCM offers a good structure to support availability requirements. For "availability" placement we assume that all edge weights $w_{l}$ are equal to one, because we want to spread instances regardless of the latest network congestion. This means that the "distance" between server racks is simply the number of edges on the shortest path on VCM graph. Similar to the previous algorithm, we consider the customer's location $U$ and the corresponding triangle on $\mathrm{VCM}$ containing $U$. We then pick one of the server racks associated with that triangle, but instead of exhausting its capacity, we place only one instance on it. We then do a breadth-first search to spread instances (Fig. 10). We start with the first server and then allocate the next server by jumping over two (or more) Delaunay edges to ensure that there is some distance between the parts of an application. If we would like to guarantee that parts of an application are going to be placed precisely $p$ cells away from each other, we could also compute the " $p$ th nearest point" Voronoi Diagram - another extension of the Voronoi Diagram described in [24].

\section{Geography, capacity, and other business constraints}

In its simplest form, a placement algorithm makes a binary decision based on whether a particular target for placement has the desired capacity. There are many reasons why cloud providers would want to modify this approach. Some targets might be in lower cost locations, managing power efficiency is one example [18]. Supply could be constrained in a particular location, causing the cloud provider to want to bias placements toward adjacent locations. The cloud provider may wish to reserve capacity in a particular geography for customers either willing to pay more or willing to make a long term commitment to use that capacity. Also, a binpacking algorithm could be applied to a collection of physical capacity to ensure that un-utilized "bubbles" do not form within the cloud. Finally, the cloud provider may wish to gradually drain capacity from a rack of servers, for example, to return at end of a lease or refresh with new technology.

VCM and application placement algorithms could be extended to satisfy those additional business requirements. Below are two examples of such algorithmic extensions.

Example 1 (Satisfying Geographic Placement Constraint) As we mentioned before, guarantees against correlated failure might require certain distance separation. While we could use rough spreading for availability, as described in Fig. 10, broader spreading algorithms may be required to ensure independence of failure modes. Also, legal and other restrictions might require constraining placement to a specified geographic area. For example, some countries require personal data of their citizens to be retained within their geographic border. While the simple spread algorithm above 
Fig. 10 Availability placement algorithm

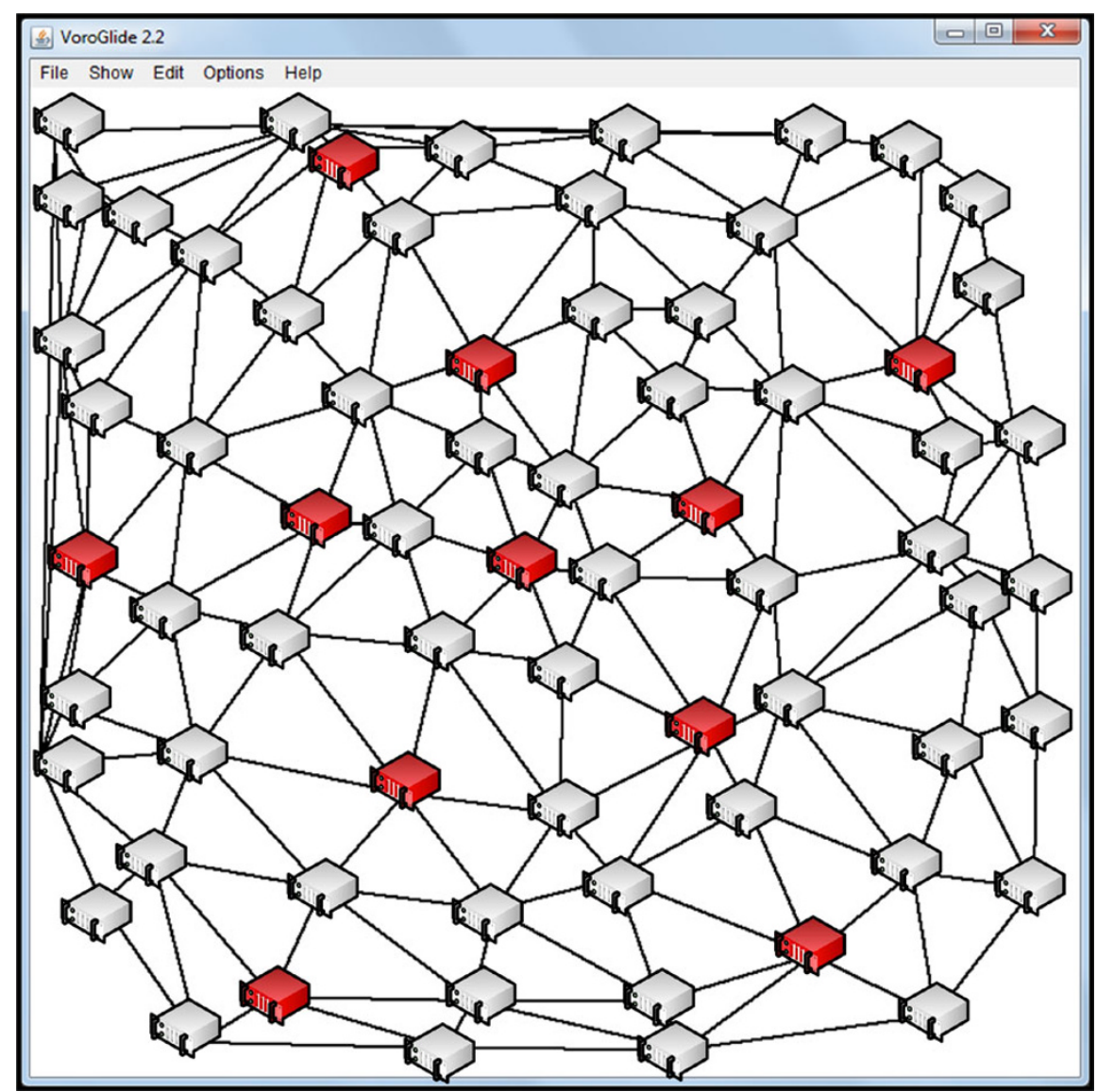

effectively scatters instances to at least $N$ Delaunay edges apart, stricter guarantees about either distance separation or location within a political boundary could require a more exact geographic constraint. Because the VCM encodes geographic location in a computationally accessible manner, an efficient addition to the placement algorithm is enabled. Instead of a general "separation" parameter, the API outlined above could include a "distance-separation" or "jurisdiction" parameter. The enhanced separation parameter would specify a minimum distance between $V M$ s activated. Instead of skipping edges for spread, as detailed previously in Fig. 10, we continue the breadth-first search to identify the next placement candidate vertex. We calculate the distance between each prior placement vertex and the candidate. If the distance satisfies the separation constraint, we place the VM, otherwise we continue the breadth-first search.

Strict guarantees on geographic placement can also be satisfied using the VCM. The Cloud Provider could define Jurisdictions across the VCM and use this information to exclude candidate placement vertices which do not meet the Jurisdiction constraint.

Example 2 (Satisfying Capacity Constraints) Cloud providers need the ability to weight placement decisions to steer capacity utilization based on the factors described above.
We are using the term "capacity" to subsume the available quantity of a range of resources that applications may require from a VM. Network I/O, CPU, Disk I/O, disk space, and memory are the most important of these though there are also special resources, such as the existence of a GPU or other special processing unit. Cloud Providers, today, are trending toward providing these in fixed ratios per VM. Allowing applications variability in their use of resources creates the potential for one application to impact the performance of another by increasing its consumption to a point of competition. Variability also makes bin-packing on physical servers far more difficult. For the purposes of our placement algorithm, however, we only suggest that the cloud provider encapsulate all of the applications' various resource needs in a single function, encapsulate the available capacity on a physical server in a similar single function, and then compare the two for placement.

A more complex version of the basic placement algorithm uses both the geometric capabilities of the VCM and replicated capacity information to favor placement candidates which are underutilized, even though they may have less optimal latency and bandwidth for the placement. There are many efficient algorithms for spreading state information across a large-scale distributed system. One example is a gossip approach [8] which allows scalable, reliable commu- 
nication of capacity utilization across the cloud. Each server, gossiping its current capacity as well as re-gossiping information on other servers it has heard about, becomes aware of the general state of capacity in all directions from the similar gossip of all other servers.

Upon being chosen as the target for assignment of a virtual machine, the server's utilization-its capacity used divided by capacity available - is compared with the average $A U$ of all gossiped utilization. If greater, the server is skipped as though it had been full and the breadth-first search continues.

1. Put all neighbors of $R_{i}$ in a list (same as before).

2. Examine all server racks in the list and select the server rack $R_{j}$ closest to $R_{i}$ using the definition of the "distance" provided above (same as before).

3. Compare the utilized capacity of $R_{j}$ with $A U$. If below $A U$, allocate capacity of $R_{j}$ to our application

4. Add all neighbors of $R_{j}$ to the list.

5. Remove $R_{j}$ from the list.

6 . Repeat steps $2-5$ until we find requested capacity to launch $k$ instances to run the application.

Rather than being calculated dynamically the utilization target could be set by the Cloud Provider explicitly. Setting a target is useful for guiding capacity or draining racks prior to maintenance or upgrade. The target could also incorporate historical analysis of existing occupants, probabilistic demand, and could lead to sophisticated decision-making on placement of the sort considered in [17].

\section{Virtual machine migration}

There are several reasons to periodically re-examine and rearrange applications:

1. Similar to disk fragmentation, over time the Computer Cloud becomes fragmented.

2. With changes in network latency, application might benefit from a new placement, which takes into account the latest network latency (stored as weights $w_{l}$ in VCM).

3. Cloud Providers might discover that several applications are expressing virtual affinity implicitly through network utilization and would benefit from being placed closer.

It is advisable to perform periodic Cloud defragmentation. Defragmentation is performed by re-arranging parts of application, which are talking through a congested area using placement algorithm described above. Given the distributed nature of Voronoi Diagram, defragmentation could be performed locally in response to network congestion and also scheduled periodically as a preemptive maintenance of the Cloud.

There has been significant research in using live migration of virtual machines to optimize resource utilization.
VM migration as described in [7] uses low-latency, highbandwidth local networks to achieve interruption times as low as $60 \mathrm{~ms}$. The notion of "capsules" in [28] provides some hope for moving VMs over longer distances. A checkpointing approach is used in [14] to specifically tackle the problem of migration over longer distances. The problem of scheduling migrations is tackled in [30]. In addition to methods for migration, analysis on the lifetime of virtual workloads suggests that opportunity for optimization does exist. In a study of PlanetLab resources [25] 25\% of "slices" were shown to live more than 1 week, offering significant opportunity for observation and migration. Research and practice on migration of VMs shows that proximity plays an important part in the decision for placing migrations. Applications "dirty" more or less memory at different rates, driving the amount of bandwidth and time required to sync a new VM, influencing the constraint on distance to the new location. Tolerance for the duration of interruption also influences the optimal choice of a distance constraint. Current research does not suggest how VM proximity should be managed. The VCM's ability to consider distance in every placement makes it especially suited to solving the migration problem, both to minimize downtime for the application and to enable a wider geographic choice of placement.

Using a working example of vacating a rack of servers $R_{i}$ to allow replacement or retirement, a list of VMs hosted on $R_{i}$ which require relocation is established. This list contains the capacity requirement of the $\mathrm{VM} C_{v}$ and the location of its user $U_{v}$. For each member of the list, the aforementioned Latency Constraint placement algorithm is performed:

1. Find the triangle $T$ in the Delaunay triangulation containing $U_{v}$.

2. Identify the three vertices of $T$, select the closest vertex to $U_{v}$.

3. If the server rack at this vertex $R_{j}$ has capacity $C_{v}$, execute migration of the VM to $R_{j}$, process next member of the list.

4. If not, put all neighbors of $R_{j}$ in a list.

5. Examine all server racks in the list and select the server rack $R_{j}$ closest to $R_{i}$ using the definition of the "distance" provided above (as the sum of all weights on the shortest path).

6. If $R_{j}$ has available capacity then allocate available capacity of $R_{j}$ to our application.

7. Add all neighbors of $R_{j}$ to the list.

8. Remove $R_{j}$ from the list.

9. These steps are repeated until all VMs on the list have been migrated.

Optionally, a pre-sort of the migration list could be performed to ensure the best user outcome. For example, the simplest sort could be by network utilization, giving first 
priority to VMs with the heaviest dependency on the network. Additionally, the list could be pre-sorted by $U$, ensuring that an individual user's VMs were placed and migrated as a group, maximizing their chance of maintaining the closest possible distance between them.

\section{Conclusions and future work}

In our study we propose to adapt a well-known structure, the Voronoi Diagram, to modeling Computer Clouds. We present a novel Virtual Cloud Model-a combination of static geographical Delaunay Triangulation, supplemented with near-real-time network latency information. We describe one algorithm for placing "low-latency" applications and another algorithm for placing "high-availability" applications in the Computer Cloud using VCM. It appears to be the first application of Voronoi Diagrams to managing the assignment of applications to physical servers in the Cloud. The algorithm is scalable and easily localizable and distributable, which is instrumental in managing tens of millions of applications running on millions of servers, located across the world. The proposed system can be used as a part of a larger system which can take all of the user parameters into account and suggest placement strategies. In our discussion below on future research we highlight a few of these areas, specifically Virtual Machine Migration, Bandwidth Weighting and Group Placement Constraints.

\subsection{Bandwidth weighting}

Up to now, we have considered applications for which latency is the most significant network consideration. While latency generally correlates with bandwidth [25] it is possible to construct networks and applications optimized for bandwidth over latency. For these applications we propose carrying dual edge weights, one for latency and the other for bandwidth. While multiple VCM edge weights would be problematic in the case where a given placement would require consideration of all weights simultaneously, the highbandwidth high-delay network requirement could be satisfied with such a scheme.

\subsection{Group placement constraints}

Though default placement considers all members of a group of virtual machines in a placement request to have equal connectivity requirements with respect to each other, many distributed applications have components which may require specific network affinity to other components. Consider a web application made up of many web servers each tied to a specific memory cache server, where session affinity desires low latency between specific pairs of web and cache servers. The simplest form of our placement algorithm, described above, could be modified to allow group placement through successive calls, favoring closeness of specific virtual machines to other, previously placed virtual machines. If the $U$ location parameter is set equal to a previous $R_{i}$ which received a placement, the subsequent call will attempt to place the new virtual machine as close as possible (respecting any specification of a required availability separation, of course). However, it is easy to imagine a set of constraints which should be satisfied as a group, at the time of a single launch request for a set of virtual machines. For this case, our algorithm could be improved further by creating a constraint list declaration to be processed during the breadth-first search for rack capacity. A set of physical racks, and/or specific servers would be selected and reserved, which meet all requirements of the group's constraint list, followed by the actual placement through the reservations. This would allow the group's placement request to be processed as an all-or-none transaction, ensuring all constraints are met.

Additional applications of VCM could be the object of further research:

1. For example, VCM could be used by Cloud Providers to identify and cure inefficient routing and network topologies by examining cases where the weight $w_{l}$ of an edge is out of proportion with its length.

2. VCM could also be used for making bandwidth reservations by allocating static routes and reserving resources for Quality of Service (QoS). Some applications require predictable, guaranteed performance and VCM might offer an efficient structure to manage and prioritize Cloud resources to offer a certain guaranteed level of performance to some applications.

3. VCM could also be used to share application placement information across different Clouds. This allows customers to control application placement and run their applications close to each other, but on different Clouds offered by different providers.

4. VCM could also be used to manage Cloud Capacity. Each placement request creates demand for a particular placement. In addition to satisfying the request, we could add phantom points on the VCM graph representing optimal placement, thus indicating a need for new capacity in a region of the VCM. We would need additional information on total demand, target utilization factors, and server-rack capacity, to produce a set of candidate points near which additional capacity is recommended.

5. We could also use VCM to manage donations of excess capacity to non-for-profit organizations world-wide. By identifying portions of the cloud with less demand at certain times we could mark those portions as donation opportunities. 
Acknowledgements The second author is supported in part by the National Science Foundation (NSF) Grants DMS-0652005 and DMS0969254.

\section{References}

1. Agarwal S, Dunagan J, Jain N, Saroiu S, Wolman A, Bhogan H Volley: Automated data placement for geo-distributed cloud services. In: Proceedings of 7th USENIX symposium on networked systems design and implementation (NSDI)

2. Aurenhammer F (1991) Voronoi diagrams - a survey of a fundamental geometric data structure. ACM Comput Surv 23(3):345405

3. Bae SW, Chwa K-Y (2004) Voronoi diagrams with a transportation network on the Euclidean plane. In: Algorithms and computation: 15th international symposium, ISAAC, Hong Kong, 2004, pp 101-124

4. Beaumont $\mathrm{O}$ et al (2006) VoroNet: a scalable object network based on Voronoi tessellations. Laboratoire de l'Informatique du Parallélisme, Lyon, France, RR2006-11

5. Brakke KA (1985) Statistics of random plane Voronoi tessellations. Techn Report, Department of Math Sciences, Susquehanna University, Selinsgrove

6. Calka P (2003) An explicit expression of the distribution of the number of sides of the typical Poisson? Voronoi Cell Adv Appl Probab 35:863-870

7. Clark C et al (2005) Live migration of virtual machines. In: USENIX NSDI

8. Demers A, Gealy M, Greene D, Hauser C, Irish W, Larson J (1989) Epidemic algorithms for replicated database maintenance. Association of Computing Machinery, New York

9. Devillers O, Pion S, Teillaud M (2001) Walking in a triangulation. In: 17th annual ACM symposium on computational geometry (SCG), pp 106-114

10. Fortune S (1992) Voronoi diagrams and Delaunay triangulations. In: Hwang F, Du D-Z (eds) Computing in Euclidean geometry. World Scientific, Singapore, pp 193-233

11. Hinde AL, Miles RE (1980) Monte Carlo estimates of the distributions of the random polygons of the Voronoi tessellation with respect to a Poisson process. J Stat Comput Simul 10:205-223

12. Hu S-Y, Liao G-M (2004) Scalable peer-to-peer networked virtual environment. In: Proc ACM SIGCOMM workshop on NetGames, pp 129-133

13. Icking $C$ et al (2003) Java applets for the dynamic visualization of Voronoi diagrams. In: Lecture notes in computer science, pp 191-205

14. Jin H, Liu H, Liao X, Hu L, Li P (2009) Live migration of virtual machine based on full-system trace and replay. In: HPDC '09 proceedings of the 18th ACM international symposium on high performance distributed computing. ACM, New York

15. Khanban A, Edalat A, Lieutier A et al (2002) Computability of partial Delaunay triangulation and Voronoi diagram. In: Computability and complexity in analysis, CCA 2002, July 2002
16. Kozuch M, Gass R, Ryan M (2009) Tashi: Location-aware cluster management. In: International conference on autonomic computing. ACM, New York, pp 43-48

17. Kramer L, Barbulescu L, Smith S Understanding performance tradeoffs in algorithms for solving oversubscribed scheduling. The Robotics Institute, Carnegie Mellon University

18. Le K, Zhang J, Meng J, Bianchini R, Nguyen T, Jaluria Y (2010) Reducing electricity cost through virtual machine placement in high performance computing clouds. Technical Report DCS-TR680, Dept of Computer Science, Rutgers University, November 2010

19. Lee D, Lam SS (2008) Efficient and accurate protocols for distributed Delaunay triangulation under churn. In: Network protocols, ICNP 2008, pp 124-136

20. Meijering JL (1953) Interface area, edge length, and number of vertices in crystal aggregates with random nucleation. Philips Res Rep 8:270-290

21. Meyerhenke H (2005) Constructing higher-order Voronoi diagrams in parallel. In: EWCG, pp 123-126

22. Møller J (1994) Lectures on random Voronoi tessellations. Springer, New York

23. Na H-S, Lee C-N, Cheong O (2002) Voronoi diagrams on the sphere. In: Computational geometry: theory and applications, September 2002, pp 183-194

24. Okabe A, Boots B, Sugihara K (1992) Spatial tessellations: concepts and applications of Voronoi diagrams. Wiley, Chichester

25. Oppenheimer D, Chun B, Patterson D, Snoeren A, Vahdat A (2006) Service placement in a shared wide-area platform. In: ATEC '06 proceedings of the annual conference on USENIX '06 annual technical conference. USENIX Association, Berkeley

26. Ostrobsky-Berman Y (2005) Computing transportation Voronoi diagram in optimal time. In: EWCG, pp 159-162

27. Ostrovsky-Berman Y (2003) Transportation Voronoi diagrams. Leibniz Center for Research in Computer Science, Jerusalem

28. Sapuntzakis C, Chandra R, Pfaff B, Chow J, Lam S, Rosenblum M (2002) Optimizing the migration of virtual computers. In: Proceedings of the 5th symposium on operating systems design and implementation (OSDI'02), December 8-11, 2002, Boston, MA, USA

29. Sedgewick R (1990) Algorithms in C. Addison-Wesley, Reading

30. Setzer T, Stage A (2009) Network-aware migration control and scheduling of differentiated virtual machine workloads. In: CLOUD '09 proceedings of the 2009 ICSE workshop on software engineering challenges of cloud computing. IEEE Computer Society, Washington

31. Shamos M, Hoey D (1975) Closest-point problems. In: Proc 16th annu IEEE sympos found comput sci, pp 151-162

32. Sriram I, Khajeh-Hosseini A (2010) Research agenda in cloud technologies. In: 1st ACM symposium on cloud computing, SOCC. ACM, New York

33. Tanemura M (2003) Statistical distributions of Poisson Voronoi cells in two and three dimensions. Forma 18(4):221-247

34. VoroGlide (2010) Interactive Voronoi diagrams. Cooperative systems [Online]. http://www.pi6.fernuni-hagen.de/GeomLab/ VoroGlide/ 